\title{
An Experimental Evidence of Asymmetrical Distribution of the Electromagnetic Field on Transcranial Magnetic Stimulation Applications
}

\author{
Francisco Mesa Linares ${ }^{1 *}$ and Laura Cruz Vazquez ${ }^{2}$ \\ =Autonomous University of Baja California, Mexico \\ ${ }^{2}$ Department of Processes, Technological University of Tijuana, México
}

*Corresponding author: Francisco Mesa Linares, School of Technology and Engineering Sciences, Autonomous University of Baja California, Postal code: 21500, Tijuana, Baja California, Mexico

Submission: 海 February 15, 2018; Published: 制 February 28, 2018

\begin{abstract}
Transcranial Magnetic Stimulation is a therapeutic technique associated with the diagnosis and therapeutic applications of the central nervous system disorders. Parkinson's disease, depression, schizophrenia and Neuropsychiatry are some of the disease that is commonly treated with TMS. Although this technique has been widely approved for clinical use, relatively little information is recorded in the literature associated with their optimal parameters of the application. Field focalization, electromagnetic field distribution, coil orientation and current direction correspond to some of these criteria. Also, most mathematical and computational studies, for example, are usually based on initial conditions that assuming a symmetrical electromagnetic field distribution. An array of parallel straight wires was used to measure the inductive effect. The array was placed in parallel planes to the transverse area of the coil, and at various orientations. Preliminary results have shown an asymmetrical distribution associated with the electromagnetic field induced. Such interpretation could provide relevant information about the project and the direction of the induced current for therapeutic applications.
\end{abstract}

Keywords: Central nervous system; TMS; Electromagnetic field; Magnetic stimulation; Circular coil

\section{Introduction}

Transcranial magnetic Stimulation (TMS) is a therapeutic technique focused on central nervous system disorders [1]. Essentially, TMS consists in stimulating excitable brain cells by electrical current electromagnetic field-induced. The electromagnetic field can penetrate without perceptible attenuation through the skin, subcutaneous and bone tissue, inducing electric current inside the brain, even without physical contact with the source [2]. Based on the Biot-Savart's law, Faraday's induction and Maxwell Equations in general, this technique includes application of level of electromagnetic fields with sufficient magnitude to depolarize neurons and modulate cortical excitability [3]. A magnetic stimulator basically consists in a voltage source, and a capacitive array which produce an electric current that moves across an inductive coil. Such current is adjusted to achieve the required stimulation [4].

The first TMS experiments were performed by D'Arsonval in the year of 1896 [5] whom reported the presence of phosphenes in response to stimulating his own brain using a huge motor. However, it was until 1985 when A. T. Barker and colleagues [6] stimulated the human motor primary cortex as well as peripheral nerves. The Barker's stimulator delivered single pulses at frequencies below
$1 \mathrm{~Hz}$. Higher frequencies did not allow complete charge of the capacitors and overheated the coil. By 1987 new stimulators were built, enhanced by the capacity to work at higher frequencies [7]. Nowadays, researchers are working on new ways that explain the cell membrane functioning using magnetic stimulation coils [8] and TMS is currently being used by researchers interested in diverse clinical fields. Pioneering therapeutics works were developed on Parkinson's disease [9] depression [10] schizophrenia [11] and neuropsychiatry [12]. For clinical purposes, the most important TMS parameters are basically four: the focalization degree, distance of application, pulse intensity and frequency [13] which are determined by the particular stimulation setup. More earlies research has been centered about the repetitive transcranial magnetic stimulation (rTMS) modalities [14,15] TMS is a therapeutic technique that has been approved for clinical use; however, some important criteria and physical characteristic, such as the knowledge about the induced field distribution, focus area, optimal stimulation frequency and intensity, constitute areas of investigation very little explored. The study of these characteristics composes an interesting area that would allow understanding in a better way the biological systems electrical behavior $[16,17]$. 
Although there have been efforts to achieve a higher level of specificity through imaging techniques [18] it is necessary to provide more information related to the spatial distribution of the electromagnetic field during the real application of the TMS technique. Considering the identified areas of opportunities, and with the intention of provide information respect the characterization of the electromagnetic field applied in TMS protocols, this short report include an experimental evaluation about the spatial distribution of the electromagnetic field generated with a Magstim 220 electromagnetic stimulator (The Magstim Company Ltd, Whitland, and South West Wales) with $9 \mathrm{~cm}$ circular coil inner diameter, capable of producing 2 Tesla like maximum electromagnetic field. The voltage induced in a No. 22 AWG (29.1 $\mathrm{mm}$ outer diameter) wire array with $40 \mathrm{~cm}$ length and spaced to fixed distance of $1 \mathrm{~cm}$, was measured, using a $100 \mathrm{MHz}$ bandwidth digital oscilloscope (TDS-3032b, Tektronix Inc. Beaverton OR).The voltage induced for $50 \%$ of electric charge capacity in the stimulator was measured with the wire array, placed at different distances from the stimulation coil. Preliminary results suggest that the variation of the field in the zone immediately underneath the coil is small, and describe a no symmetrical distribution comportment respect to the geometrical coil.

The interpretation of a no symmetrical comportment of the electromagnetic field constitutes a novelty. Most mathematical and computational studies about current induced throughout TMS applications in cerebral cortex are typically based on initial conditions that assuming symmetrical electromagnetic field distribution [19-21]. In such representative reports and similar, could be omitting any other effect beyond the geometric configuration of the coil or arrangement of coils. The asymmetry could be due to the magnetic field produced by the feeder cable of the coil (handle), which contributes to the total magnetic field. Also, earliest evaluations show a possible dependence of the orientation of a detector configuration, and suggest that when applying TMS, it is important to consider the position of the coil applied to the stimulated zone, so the intensity generated is maximal, and stimulus dispersion is minimal.

When electrical current is applied to the nervous system, it has been recommended the use of small intensities [22]. In this sense, the present short reflection suggests that when TMS is applied, it is important to consider that optimal stimulation could be affected by the coil's arm orientation. The commercial magnetic stimulator commonly is presented in the form of a coil, or two coils connected, in order to achieve greater focuses on the field. In both cases, symmetrical field distribution is usually considered in clinical applications. Our preliminary experimental results could provide relevant information about the projection and the direction of the induced current during clinical applications.

\section{Acknowledgment}

The authors express their thanks to Dr. Sergio Márquez Division of Health Sciences, University of Guanajuato; Dr. Modesto Sosa, Division of Science and Engineering, University of Guanajuato, and Dr. Miriam Astacio, Autonomous University of Baja California, for the financial support, access to the equipment, suggestions and support in general, necessary for conducting the experimental evaluations that support the report.

\section{References}

1. Camprodon JA, Pascual-Leone A (2016) Multimodal applications of transcranial magnetic stimulation for circuit-based psychiatry. JAMA Psychiatry 73(4): 407-408.

2. Klein M, Treister R, Raij T, Pascual-Leone A, Park L, et al. (2015) Transcranial magnetic stimulation (tms) of the brain: guidelines for pain treatment research. Pain 156(9): 1601-1614.

3. Rossi S, Hallett M, Rossini P, Pascual-Leone A (2009) Safety, ethical considerations, and application guidelines for the use of transcranial magnetic stimulation in clinical practice and research. Clin Neurophysiol 120(12): 2008-2039.

4. Deng ZD, Lisanby SH, Peterchev AV (2015) Effect of anatomical variability on electric field characteristics of electroconvulsive therapy and magnetic seizure therapy: A parametric modeling study. IEEE Trans Neural Syst Rehabil Eng 23(1): 22-31.

5. D'Arsonval A (1896) Dispositifs pour la mesure des courants alternatifs de toutesfréquences. CR SociétéBiologique 3: 450-457.

6. Barker A, Jalinous R, Freeston I (1985) Non-invasive magnetic stimulation of the motor cortex. Lancet 1(8437): 1106-1107.

7. Pascual-Leone A, Valls-Sole J, Wassermann E, Hallett M (1994) Responses to rapid-rate transcranial magnetic stimulation of the human motor cortex. Brain 117 (4): 847-858.

8. Davey K, Epstein C (2000) Magnetic stimulation coil and circuit design. IEEE Trans Biomed Eng 47(11): 1493-1499.

9. Pascual-Leone A, Alonso M (1995) Lasting beneficial effect of rapid-rate transcranial magnetic stimulation on slowness in Parkinson's disease. Neurology 45(4): A315

10. George M, Wassermann E, Krimbell T, Little JT, Williams WE, et al. (1997) Mood improvement following daily left prefrontal repetitive transcranial magnetic stimulation in patients with depression: a placebo-controlled crossover trial. Am J Psychiatry 154(12): 1752-1756.

11. Geller V, Grisaru N, Abarbanel J, Lemberg T, Belmaker RH (1997) Slow magnetic stimulation of prefrontal cortex in depression and schizophrenia. Prog Neuropsychopharmacol Biol Psychiatry 21(1): 105-110.

12. Pridmore S, Belmaker R (1999) Transcranial magnetic stimulation in the treatment of psychiatric disorders. Psychiatry Clinical Neurosciences 53(5): 541-548.

13. Nadeem M, Thorlin T, Gandhi O, Persson M (2003) Computation of electric and magnetic stimulation in human head using the 3-D impedance method. IEEE Trans Biomed Eng 50(7): 900-907.

14. Jean-Pascal L, André-Obadia N, Antal A, Ayache SS, Baeken C, et al. (2014) Evidence-based guidelines on the therapeutic use of repetitive transcranial magnetic stimulation (rTMS). Clinical Neurophysiol 125(11): 21502206.

15. Neville LS, Hayashi CY, Alves S, Zaninotto AL, Sabino JP, et al. (2015) Repetitive Transcranial Magnetic Stimulation (rTMS) for the cognitive rehabilitation of traumatic brain injury (TBI) victims: study protocol for a randomized controlled trial. Trials 16: 440 .

16. Andreou AP, Holland PR, Akerman S, Summ O, Fredrick J, et al. (2016) Transcranial magnetic stimulation and potential cortical and trigeminothalamic mechanisms in migraine. Brain 139(1): 2002-2014.

17. Barker AT, (2017) Transcranial Magnetic Stimulation-past, present and future. Brain Stimulation 10(2): 346-540. 
18. Kobayashi M, Pascual-Leone A (2003) Transcranial magnetic stimulation in neurology. Lancet Neurol 2(3):145-156.

19. Chervyakov AV, Chernyavsky AY, Sinitsyn DO, Piradov MA (2015) Possible mechanisms underlying the therapeutic effects of transcranial magnetic stimulation. Front Hum Neurosci 9(303): 1-14.

20. Kozyreva V, Eyselb UT, Janckea D (2014) Voltage-sensitive dye imaging of transcranial magnetic stimulation-induced intracortical dynamics. Proc Natl Acad Sci USA 111(37): 13553-13558.
21. Paffi A, Camera F, Carducci F, Rubino G, Tampieri P, et al. (2015) A Computational Model for Real-Time Calculation of Electric Field due to Transcranial Magnetic Stimulation in Clinics. International Journal of Antennas and Propagation, pp. 1-11.

22. Collado-Corona M, Mora-Magaña I, Cordero-Guzmán L (2000) Estimulación magnética transcranealy trauma acústico. CirCiruj 68(1): 10-14. (c) (i) Creative Commons Attribution 4.0 International License

For possible submissions Click Here

\section{Submit Article}

EPMR
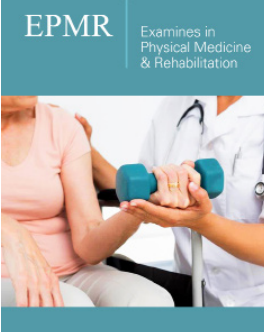

Examines in Physical Medicine and Rehabilitation: Open Access

\section{Benefits of Publishing with us}

- High-level peer review and editorial services

- Freely accessible online immediately upon publication

- Authors retain the copyright to their work

- Licensing it under a Creative Commons license

- Visibility through different online platforms 